\title{
TIM-4 promotes the growth of non-small-cell lung cancer in a RGD motif-dependent manner
}

\author{
Qianqian Zhang ${ }^{1,2,4}$, Hongxing Wang ${ }^{1,4}$, Xiaodong Wu ${ }^{1,3}$, Bing Liu ${ }^{1}$, Wen Liu ${ }^{1}$, Rong Wang ${ }^{1}$, Xiaohong Liang ${ }^{1}$, \\ Chunhong $\mathrm{Ma}^{1}$ and Lifen Gao*,1 \\ ${ }^{1}$ Department of Immunology, Key Laboratory for Experimental Teratology of Ministry of Education, Shandong Provincial Key \\ Laboratory of Infection \& Immunology, Shandong University School of Medicine, 44 Wenhua Xi Road, Jinan, Shandong 250012, \\ People's Republic of China; ${ }^{2}$ Institute of Cardiovascular Disease, General Hospital of Ji'nan Military Region, 8 Lashan Road, Ji'nan, \\ Shandong 250022, People's Republic of China and 'Department of Packaging, Jinan Blood Centre, 127 Jing Qi Road, Jinan, \\ Shandong 250021, People's Republic of China
}

Background: T-cell immunoglobulin domain and mucin domain 4 (TIM-4) is exclusively expressed in antigen-presenting cells and involved in immune regulation. However, the role of TIM-4 expressed in tumour cells remains completely unknown.

\begin{abstract}
Methods: Immunohistochemistry staining was used to examine TIM-4 or Ki-67 expression in tumour tissues. Real-time PCR or RT-PCR was performed to detect TIM-4 mRNA expression. Lung cancer cell growth and proliferation were conducted by CCK-8 assay and EdU staining. Cell cycle progression was analysed by flow cytometry. The PCNA and cell cycle-related proteins were verified by western blot. Co-IP assay was used to identify the interaction of TIM- 4 and integrin $\alpha v \beta 3$. The efficacy of TIM- 4 in vivo was evaluated using xenograft tumour model.
\end{abstract}

Results: The expression of TIM-4 in non-small-cell lung cancer (NSCLC) tissues was significantly higher than that of the adjacent tissues. Enhanced TIM-4 expression was negatively correlated with histological differentiation of lung carcinoma and lifespan of patients. Overexpression of TIM-4 promoted lung cancer cell growth and proliferation, and upregulated the expression of PCNA, cyclin A, cyclin B1 and cyclin D1, accompanied by accumulation of lung cancer cells in S phase. Interestingly, Arg-Gly-Asp (RGD) motif mutation abolished the effect of TIM-4 on lung cancer cells, which was further verified by tumour xenografts in mice. Furthermore, we found that TIM-4 interacted with $\alpha \mathrm{v} \beta 3$ integrin through RGD motif.

Conclusions: This finding suggests that TIM-4 might be a potential biomarker for NSCLC that promotes lung cancer progression by RGD motif.

Lung cancer is one of the most common malignant tumours. Emerging evidences show that lung cancer has become the leading cause of cancer deaths in both males and females worldwide (Siegel et al, 2014). Non-small-cell lung cancer (NSCLC) is the most common type of lung cancer, accounting for $\sim 85 \%$ of all cases of lung cancer. Most NSCLC patients present with advanced tumours that result in poor prognosis. Smoking, respiratory system disease and environmental factors are known risk factors of lung cancer pathogenesis. However, the development of lung cancer is an extremely complicated process in which multiple genes, including genes encoding immune molecules such as B7-H1, B7-H3 and B7H4, are involved (Chen et al, 2012; Boland et al, 2013; Velcheti et al, 2014). Therefore, it is urgent to identify the important molecular regulators for lung cancer in order to introduce the novel therapeutic strategies.

The T-cell immunoglobulin domain and mucin domain (TIM) family is a relatively newly discovered group of molecules and has received increasing attention because of its important roles in

\footnotetext{
*Correspondence: Dr L Gao; E-mail: glfflg@sdu.edu.cn

${ }^{4}$ These authors contributed equally to this work.
}

Received 26 March 2015; revised 29 June 2015; accepted 14 August 2015; published online 29 October 2015 
asthma, allergy and autoimmunity (Li et al, 2013c). In human subjects, the TIM family contains three members: TIM-1, -3 and -4 . The TIM proteins belong to type-I cell-surface glycoproteins composed of a signal peptide, an extracellular $\operatorname{IgV}$ domain, a mucin-like domain, a transmembrane domain and an intracellular cytoplasmic tail. In contrast to TIM-1 and TIM-3, TIM-4 contains a conserved arginine-glycine-aspartic acid (RGD) motif in $\mathrm{IgV}$ domain that is the ligand of integrins. In addition, TIM-4 expression is highly restricted to professional antigen-presenting cells (APCs), suggesting that TIM-4 might exert an important role in regulating immune functions (Meyers et al, 2005). It has been found that TIM-4 is essential for the maintenance of the homeostatic state of resident peritoneal macrophages and plays a dual role in the induction and effector phases of murine arthritis (Wong et al, 2010; Abe et al, 2013). Our published data also show that TIM-4 negatively regulates the function of macrophages (Xu et al, 2010). Though it was originally thought that TIM-4 is not expressed in other immunocytes except APCs, (Kim et al, 2010 and Toda et al, 2012) reported the expression of TIM-4 on B1 cells and NKT cells recently. Interestingly, TIM- 4 could also be detected in the histiocytic sarcoma, histiocytic and dendritic cell neoplasms as well as Langerhans cell sarcoma (Dorfman et al, 2010; Li et al, 2013b). In addition, a case report showed that TIM-4 is expressed in parapharyngeal liposarcoma ( $\mathrm{Li}$ et al, 2013a). These studies suggested the potential role of TIM-4 in tumour development and progression. However, its expression pattern and biological functions in lung cancer remain unclear.

Here we investigated the role of TIM-4 in lung cancer progression. We showed that the expression of TIM-4 in lung cancer tissues was significantly higher than that of adjacent tissues and closely related with histological differentiation of lung carcinoma and lifespan of lung cancer patients. Importantly, TIM-4 overexpression promoted lung cancer cell growth, proliferation and accumulation in $\mathrm{S}$ phase that was performed by its RGD motif in IgV domain. Furthermore, we found that TIM-4 interacted with integrin $\alpha \mathrm{v} \beta 3$ through RGD motif that might account for the effects of TIM-4 in promoting lung cancer progression. This work presented here suggests TIM-4 as a poor prognostic indicator for NSCLC patients.

\section{MATERIALS AND METHODS}

Patient specimens. Seventy pairs of NSCLC tumour tissues and adjacent nontumour tissues (at least $3 \mathrm{~cm}$ away from the tumour site) were collected from Shandong Provincial Hospital and smallcell lung cancer patients were excluded. None of the patients had received chemotherapy or radiation before resection and all patients were followed-up after operation till 60 months. Informed consent was obtained from all patients before the study was initiated with the approval of the Shandong University Medical Ethics Committee in accordance with the Declaration of Helsinki. All data of the human subjects are summarised in Table 1. Multiple organ tumour tissue microarray (HOrg-C120PG-01) were purchased from ZuoCheng Bio-tech (Shanghai, China).

Animals. Male BALB/c nude mice (6-8 weeks of age) were purchased from Vital River Laboratory Animal Technology Co. Ltd (Beijing, China). All mice were housed in the animal facilities under the specific pathogen-free conditions. This study has been approved by the Animal Care and Use Committee of Shandong University.

The $5 \times 10^{7}$ A549 cells in $100 \mu \mathrm{l}$ phosphate-buffered saline (PBS) were subcutaneously injected into the left axillae of the nude mice and these mice were divided into three groups randomly. Tumours were injected with the indicated plasmids $(20 \mu \mathrm{g}$ per $100 \mu \mathrm{l}$ in PBS) every fourth day for a total of 4 injections after
Table 1. The expression of T-cell immunoglobulin domain and mucin domain 4 (TIM-4) in lung cancer correlates with clinical features

\begin{tabular}{|c|c|c|c|c|c|c|}
\hline & \multicolumn{6}{|c|}{$\begin{array}{l}\text { TIM-4 expression in } \\
\text { cancer tissues }\end{array}$} \\
\hline Clinical characteristics & Cases & - & + & ++ & +++ & $P$-value \\
\hline \multicolumn{7}{|l|}{ Age } \\
\hline$\geqslant 60$ & 34 & 2 & 7 & 11 & 14 & \multirow[t]{2}{*}{0.684} \\
\hline$<60$ & 36 & 5 & 8 & 11 & 12 & \\
\hline \multicolumn{7}{|l|}{ Sex } \\
\hline Male & 57 & 5 & 11 & 21 & 20 & \multirow[t]{2}{*}{0.158} \\
\hline Female & 13 & 2 & 4 & 1 & 6 & \\
\hline \multicolumn{7}{|l|}{ Pathological type } \\
\hline Adenocarcinoma & 31 & 5 & 5 & 9 & 12 & \multirow[t]{2}{*}{0.392} \\
\hline Squamous carcinoma & 39 & 2 & 10 & 13 & 14 & \\
\hline \multicolumn{7}{|l|}{ Differentiation grade } \\
\hline Poor & 24 & 1 & 2 & 6 & 15 & \multirow[t]{2}{*}{0.01} \\
\hline Well & 46 & 6 & 13 & 16 & 11 & \\
\hline \multicolumn{7}{|l|}{ Size } \\
\hline$>3 \mathrm{~cm}$ & 42 & 3 & 10 & 12 & 17 & \multirow[t]{2}{*}{0.635} \\
\hline$\leqslant 3 \mathrm{~cm}$ & 28 & 4 & 5 & 10 & 9 & \\
\hline \multicolumn{7}{|l|}{ Lymph node metastasis } \\
\hline Yes & 37 & 3 & 7 & 12 & 15 & \multirow[t]{2}{*}{0.92} \\
\hline No & 33 & 4 & 8 & 10 & 11 & \\
\hline \multicolumn{7}{|l|}{ Clinical stage } \\
\hline I & 26 & 4 & 5 & 7 & 9 & \multirow[t]{4}{*}{0.108} \\
\hline II & 17 & 2 & 7 & 6 & 2 & \\
\hline Illa & 15 & 1 & 2 & 5 & 7 & \\
\hline IIIb IV & 13 & 0 & 1 & 4 & 8 & \\
\hline
\end{tabular}

reaching a diameter of $0.5 \mathrm{~cm}$. Meanwhile, tumour size was monitored every other day. Tumour volume was calculated using the following formula: volume $=$ width $^{2} \times$ length $\times 1 / 2$ and growth curve was drawn subsequently. At 14 days after plasmid administration, the mice were killed and the tumours were isolated and weighed. Animal experiments were repeated at least twice. The TIM-4 mRNA expression in tumour xenografts was detected by real-time PCR. The TIM-4 protein expression and proliferation of tumour cells in tumour tissues were assayed by immunohistochemical staining.

Cell lines and plasmids. The human NSCLC cell lines A549, NCIH446, NCI-H1975, NCI-H1299, NCI-H358, SPCA-1 and 95-D and human monocyte cell line THP-1 were purchased from the Shanghai Cell Collection (Chinese Academy of Sciences, Shanghai, China). The A549, NCI-H446, NCI-H1299 and SPCA-1 cells were grown in Dulbecco's modified Eagle's medium (DMEM; GIBCO, Grand Island, NY, USA) supplemented with $10 \%$ fetal bovine serum (FBS; Gibco-BRL, Grand Island, NY, USA), $100 \mathrm{U} \mathrm{ml}^{-1}$ penicillin and $100 \mu \mathrm{g} \mathrm{ml}^{-1}$ streptomycin. The NCI-H1975, NCIH358, 95-D and THP-1 cells were grown in RPMI-1640 (GIBCO) supplemented with $10 \%$ FBS (Gibco-BRL), $100 \mathrm{U} \mathrm{ml}^{-1}$ penicillin and $100 \mu \mathrm{g} \mathrm{ml}^{-1}$ streptomycin. All the cells were incubated at $37^{\circ} \mathrm{C}$ in a $5 \% \mathrm{CO}_{2}$ humidified incubator.

The recombinant plasmid of full-length TIM-4 with a carboxyterminal haemagglutinin (HA) tag (pc3-hT4) was generated by PCR amplification of pcDNA3.0-hTim-4 vector with forward (5'-CCGGAATTCGATGTCCAAAGAACCTCTCATT-3') and reverse (5'-GCTCTAGACTAAGCGTAGTCTGGTACGTCG TAAGGGTAGAGGGTAAAAAGGCCGTCT- $3^{\prime}$ ) primers, where underlined nucleotides represent the sequence encoding the HA peptide tag fused in frame with TIM-4. Plasmid pc3.0-hT4(m) with RGD motif mutation into alanine was generated by PCR amplification with forward primer (5'-GCAGCTGCTGTCTCCTT GACCATCTTAAACCCCA-3') and reverse primer (5'-CGGGA 
TAGTCCCCTGAAGTCTATAT-3'), where underlined nucleotides represent the sequence encoding RGD motif mutated into alanines. pc3-hT4 plasmid was used as template to perform PCR by a KODPlus-Mutagenesis Kit (Code No. SMK-101; TOYOBO CO., LTD, Life Science Department, Osaka, Japan).

Transient transfection. A549 cells or NCI-H1975 cells were seeded at $1 \times 10^{5}$ per $\mathrm{ml}$ in 6 -well plates $(2 \mathrm{ml}$ per well) and incubated for $12 \mathrm{~h}$. The cells were transiently transfected with plasmid DNA using Lipofectamine 2000 (Invitrogen, Beijing, China) according to the manufacturer's instructions.

RT-PCR and quantitative real-time PCR. Total RNA was isolated from THP-1 cells, lung cancer cells or tumour xenografts. The RNA quality was assessed spectrophotometrically. Complementary DNA was synthesised using reverse transcription kit (TOYOBO CO., LTD) according to the manufacturer's instructions. The mRNA expression levels of TIM-4 was detected by PCR or real-time PCR. $\beta$-Actin was selected as the internal control reference gene and normalised values were used to calculate the relative mRNA expression levels. Quantitative real-time PCR reaction was run on CFX96 Real-time PCR system (Bio-Rad, Hercules, CA, USA) using the following conditions: $95^{\circ} \mathrm{C}$ for $10 \mathrm{~min}$ (an initial denaturation step), followed by 40 cycles of $95^{\circ} \mathrm{C}$ for $15 \mathrm{~s}$ and $60^{\circ} \mathrm{C}$ for $1 \mathrm{~min}$ (annealing and extension). In addition, melting curve $\left(55-95^{\circ} \mathrm{C}\right)$ was performed at the end of each run. Gene-specific primers were as follows: TIM-4, forward (5'-ACAGG ACAGATGGATGGAATACCC- $3^{\prime}$ ) and reverse (5'-AGCCTTGTG TTTCTGCG- $\left.3^{\prime}\right) ; \beta$-actin, forward ( $5^{\prime}$-GGCATCGTGATGGACT CCG- $3^{\prime}$ ) and reverse ( $5^{\prime}$-GCTGGAAGGTGGACAGCGA- $\left.3^{\prime}\right)$. The relative expression ratios of target gene were calculated considering their amplification efficiencies.

Analysis of cell proliferation and cell cycle. Cell viability was measured using the Cell Counting Kit-8 (CCK-8, CK-04, Dojindo, Tokyo, Japan). Each experiment was repeated 3 times. For $\alpha \mathrm{v} \beta 3$ blocking assay, $25 \mu \mathrm{g} \mathrm{ml}^{-1}$ of mouse anti- $\alpha \mathrm{v} \beta 3$ (CBL544, Millipore, Billerica, MA, USA) or mouse IgG were added into A549 cells $6 \mathrm{~h}$ after transfection with pc3 or pc3-hT4 plasmid DNA (Goc et al, 2012). For cell cycle analysis, cells were collected $48 \mathrm{~h}$ after transfection, stained with propidium iodide (PI, Sigma) for $15 \mathrm{~min}$ and further assayed using Beckman Coulter Flow Cytometer (FC500, Beckman-Coulter, Fullerton, CA, USA).

Western blotting. Cells were lysed by Cell LyticäM (Sigma) and added to gel-loading buffer. After boiling for $10 \mathrm{~min}$, proteins were resolved by SDS-polyacrylamide gel electrophoresis and electroblotted onto a polyvinylidene fluoride membrane (Millipore Co., Billerica, MA, USA). Membranes were blocked with 3\% BSA and incubated with indicated antibodies. After washing in PBS containing $0.5 \%$ Tween-20, the bound primary antibody was detected with anti-mouse IgG or anti-rabbit IgG (Zhongshan Co., Beijing, China). Following washes, the antibody-bound protein was visualised by enhanced chemiluminescence. Antibodies used are as follows: anti-cyclin A (BS1084, Bioworld Technology, St Louis Park, MN, USA); anti-cyclin B1 (ab32053, Abcam, Cambridge, MA, USA); anti-cyclin D1 (2261-1, Epitomics, Burlingame, CA, USA); anti-PCNA (ab92552, Abcam); mouse-anti-HA monoclonal antibody (TA-04, Zhongshanjinqiao, Beijing, China); rabbit antiHA polyclonal antibody (51064-2-AP, Proteintech, Chicago, IL, USA); anti- $\alpha \mathrm{v} \beta 3$ (CBL544, Millipore); and anti- $\beta$-Actin (TA-09, Zhongshanjinqiao).

Co-immunoprecipitation. A549 cells were transfected with pc3hT4 or pc3-hT4(m) plasmid DNA respectively. After $24 \mathrm{~h}$, the cells were harvested and cell lysates were prepared with RIPA lysis buffer. For co-immunoprecipitation (Co-IP), total cell lysates were incubated overnight with $4 \mu \mathrm{g}$ of rabbit anti-HA polyclonal antibody (51064-2-AP, Proteintech) or mouse anti- $\alpha \mathrm{v} \beta 3$
(CBL544, Millipore) antibody and further incubated with protein A/G Sepharose for $4 \mathrm{~h}$. The complex was washed three times with cell lysis buffer, and processed for further western blot analysis.

EdU immunofluorescence staining. The A549 cells were transfected with plasmid DNA of pc3, pc3-hT4 or pc3-hT4(m). After $94 \mathrm{~h}$, the transfected cells were incubated with EdU for $2 \mathrm{~h}$. Immunofluorescence staining was performed according to standard protocols using Cell-Light EdU Apollo567 In Vitro Imaging Kit (C10310-1, Ribobio, Guangzhou, China), and then the staining results were observed under fluorescence microscope.

Immunohistochemical staining. Immunohistochemical staining was performed according to standard protocols using the following antibodies: anti-TIM-4 (ab47637; Abcam) and anti-Ki-67 (ab15580; Abcam). The TIM-4 and Ki-67 staining were reported separately according to the German semiquantitative scoring system (Han et al, 2009). Eight fields of $\sim 1000$ cells from each tumour and nontumour section were counted independently by three pathologists.

Statistical analysis. GraphPad Prism (GraphPad Software, San Diego, CA, USA) was used for data analysis. The $\chi^{2}$ test was used to analyse the statistical correlation between the clinical parameters of lung cancer and the TIM-4 expression levels in tissue sections. Student's $t$-test was applied to determine significant differences between groups. Two-way ANOVA was applied to determine significant differences between different treatments at different time points, including the growth curve of A549 cells, NCI-H1975 cells and tumour xenografts in mice. The univariate analysis of survival of NSCLC patients was analysed using the log-rank test. The $P$-values of $<0.05$ were considered to be significant.

\section{RESULTS}

Enhanced TIM-4 expression in NSCLC tissues is negatively related with the prognosis of lung cancer patients. Recently, TIM-4 was found to be expressed in tumours, including histiocytic sarcoma, histiocytic and dendritic cell neoplasms, Langerhans cell sarcoma as well as parapharyngeal liposarcoma (Dorfman et al, 2010; Li et al, 2013a,b). In order to clarify the expression pattern of TIM-4 in more kinds of tumours, we examined TIM-4 expression in a variety of tumour tissues using multiple organ tumour tissue microarray by immunohistochemical (IHC) staining. As shown in Figure 1, the levels of TIM-4 expression in oesophageal cancer, colon cancer, rectal carcinoma, pancreatic cancer, breast cancer and lung cancer tissues were higher than those of the corresponding adjacent tissues respectively, indicating the correlation between TIM-4 and tumours.

To explore the significance of TIM-4 expression in tumour development, we collected NSCLC cancer tissues and paired adjacent tissues from 70 patients who have been followed-up to ensure their survival after tumour resection. We found that TIM-4 expression was detected in both lung squamous cell carcinoma and adenocarcinoma tissues (Figure 2A). Importantly, we found TIM-4 colocalised with CK-18, a marker of lung epithelial-derived tumour cells (Supplementary Figure 1) (Ramaekers et al, 1987). Furthermore, TIM-4 expression in lung cancer tissues was significantly higher than that of the adjacent tissues (Figure 2B). More importantly, higher expression of TIM-4 in lung cancer tissues was negatively correlated with the 5-year overall survival rate of patients (Figure 2C). We further analysed the correlation of TIM-4 expression levels in NSCLC tissues with patients' clinical parameters. As shown in Table 1, TIM-4 levels were significantly negatively correlated with the differentiation of lung cancer. These results suggested that TIM-4 might be involved in NSCLC pathogenesis. 

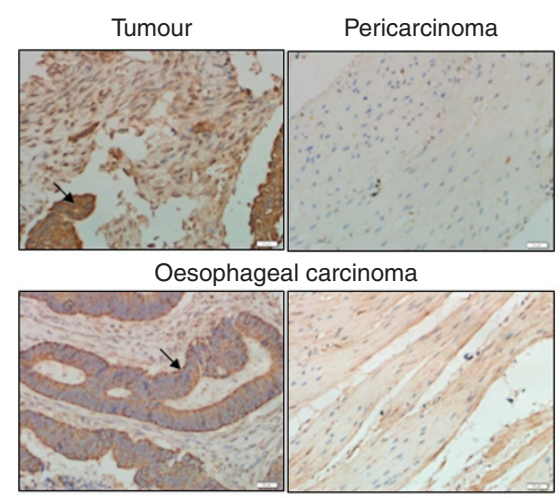

Colon cancer

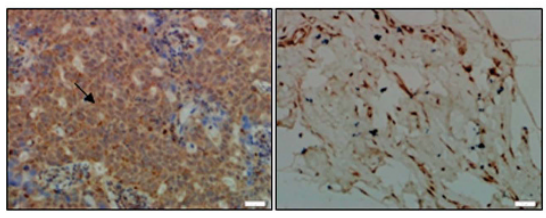

Breast cancer

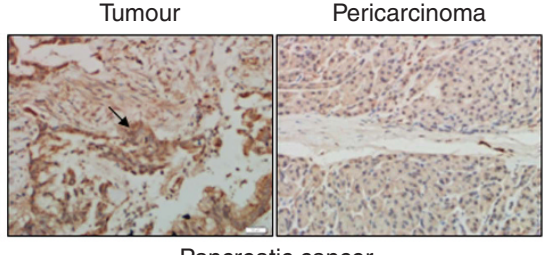

Pancreatic cancer

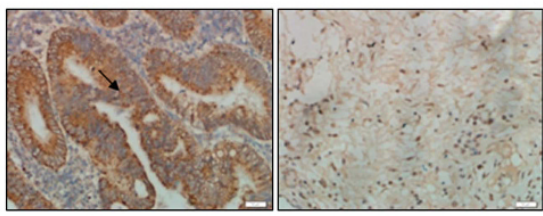

Rectal carcinoma

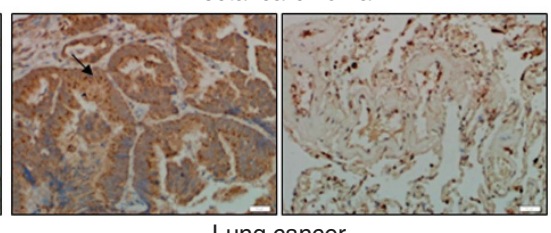

Lung cancer

Figure 1. Expression of TIM-4 in multiple organ tumour tissue microarray. Immunohistochemical (IHC) staining was performed to detect TIM-4 expression in multiple organ tumour tissue microarray. Photos of IHC staining are representative of at least 10 similar observations ( $\times 200$ ). The black arrowheads indicate the positive staining of tumour cells shown in brown.

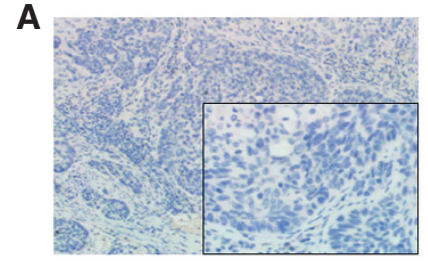

Negative control

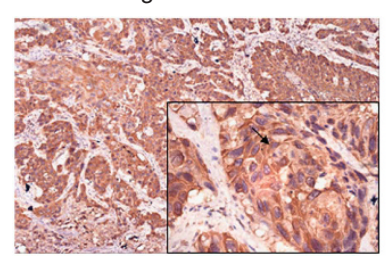

Squamous cell carcinoma
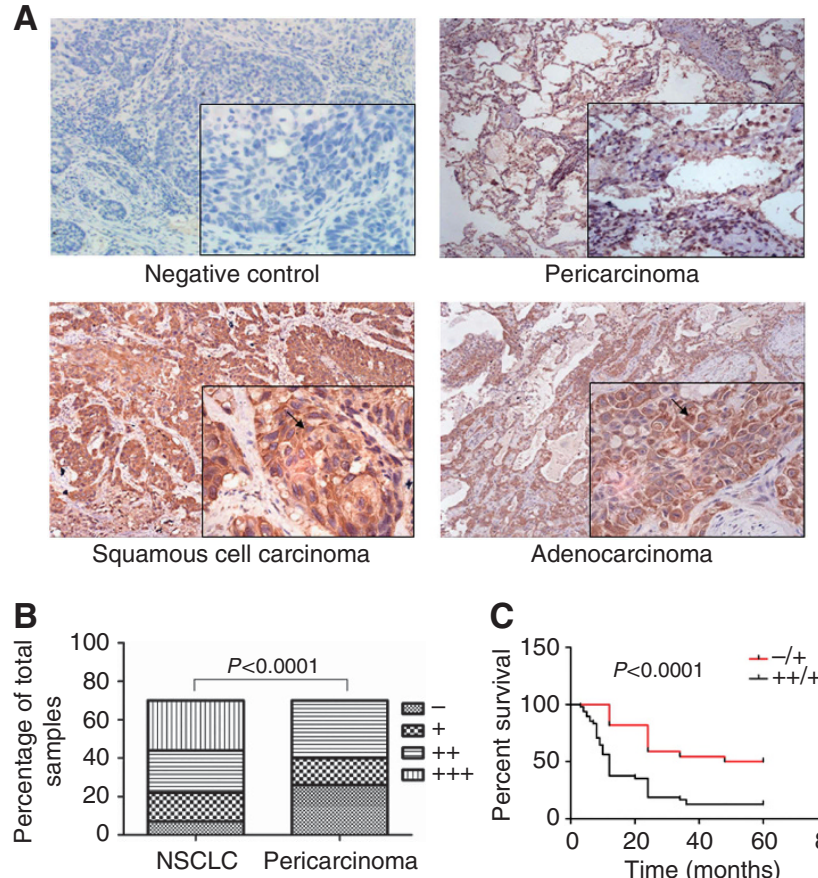

Pericarcinoma

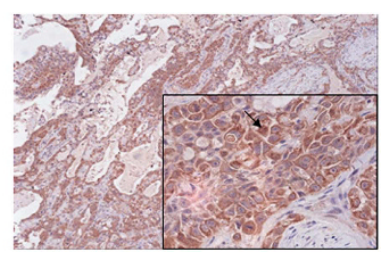

Adenocarcinoma

C

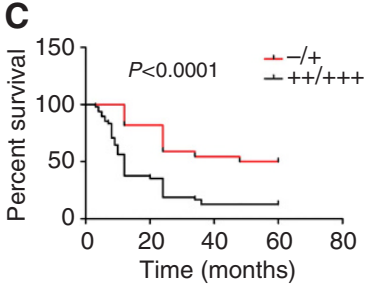

Figure 2. Enhanced TIM-4 expression in NSCLC shows poor prognosis. Immunohistochemical (IHC) staining was performed in 70 cases of NSCLC tissues and pericarcinoma tissues. (A) Representative IHC staining images of TIM-4 in NSCLC tissues and pericarcinoma issues $(\times 200)$. The negative control indicated that rabbit IgG replaced TIM-4 antibody in the process of IHC staining. (B) Staining intensity of TIM-4 in NSCLC tissues was significantly higher than that of the pericarcinoma tissues $(P<0.0001)$. (C) The survival rate of lung cancer patients with higher TIM-4 expression was significantly lower than that of the patients with lower TIM-4 expression $(P<0.0001)$.

TIM-4 overexpression promotes the growth, proliferation and cell cycle progression of lung cancer cells. As high expression of TIM-4 was found in NSCLC tissues, its exact role in lung cancer development needs to be identified in vitro and in vivo. We evaluated the TIM-4 expression levels in selected NSCLC cell line. Compared with THP-1 cells, a relatively low-level transcriptional expression of TIM-4 was detected in these lung cancer cell lines (Supplementary Figure 2A).

Our previous data showed that LPS stimulation could induce TIM-4 expression in macrophages (Xu et al, 2010). In systemic lupus erythematosus patients, TIM-4 mRNA levels in peripheral blood mononuclear cells were positively correlated with TNF- $\alpha$ level in serum, indicating that inflammatory factors might upregulate TIM-4 expression (Zhao et al, 2010). As bacterial infection and cytokines such as IL-6, TGF- $\beta$ and TNF- $\alpha$ in the inflammatory environment of tumours could accelerate the development of cancer (Tian et al, 2011), we observed the effects of LPS and these cytokines on TIM-4 expression. As shown in Supplementary Figure $2 \mathrm{~B}$ and C, stimulation with LPS, IL-6, TGF$\beta$ and TNF- $\alpha$ significantly increased TIM-4 mRNA expression in A549 and NCI-H1975 cells.

To address the role of TIM-4 in lung cancer development, human TIM-4 eukaryotic expression vector and control vector were transfected into A549 or NCI-H1975 cells respectively and the cell growth was monitored by CCK- 8 assay every day. As shown in Figures 3A and B, the growth rate of TIM-4-transfected cells was significantly higher than that of the control group, whereas knockdown of TIM-4 in A549 cells inhibited the growth of cells (Supplementary Figure 3). At $48 \mathrm{~h}$ post transfection, the cell cycle analysis was performed by PI staining and flow cytometry. Compared with the control group, there was an accumulation of cells at S phase in TIM-4-transfected A549 or NCI-H1975 cells (Figure 3C). Consistently, western blot showed that the expressions of PCNA and cell cycle-related proteins cyclin A, cyclin B1 and cyclin D1 were significantly upregulated by TIM- 4 overexpression (Figure 3D). These data suggested that TIM-4 can promote the growth, proliferation and cell cycle progression of lung cancer cells.

RGD motif is essential for TIM-4 to promote lung cancer cell proliferation and cell cycle progression. Distinct from other TIM members, there is no tyrosine phosphorylation site in the cytoplasmic region of TIM-4. However, TIM-4 molecule contains one RGD motif in IgV domain. The RGD motif in osteopontin has been shown to be responsible for tumour growth by interacting with integrin av $\beta 3$ (Cui et al, 2007). Thus, we explored the 


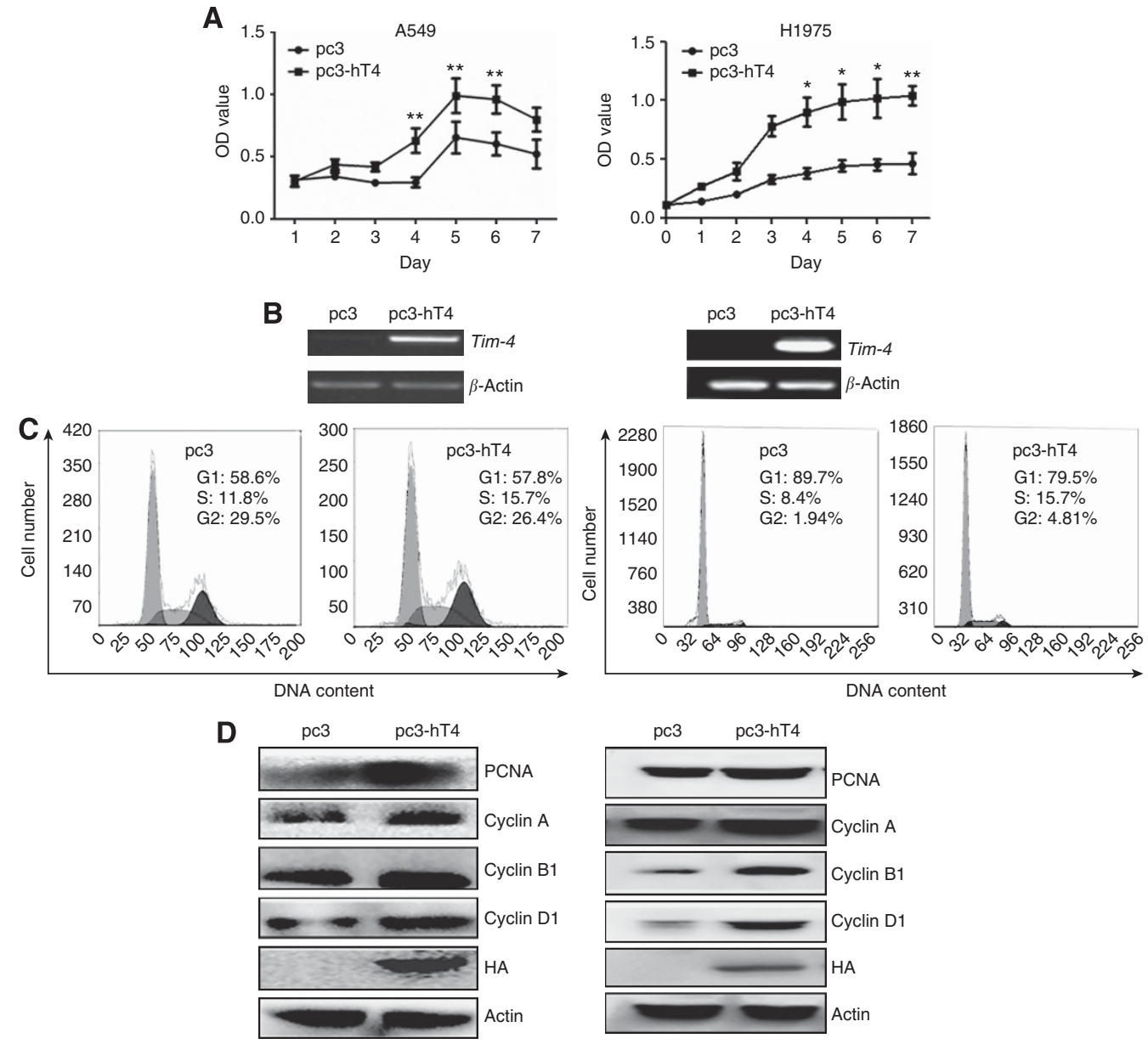

Figure 3. Overexpression of TIM-4 promotes lung cancer cell growth and cell cycle progression. Cells were set up in 96- or 6-well plates. After grown to $80-90 \%$ confluence, A549 or NCl-H1975 cells were transfected with pc3 or pc3-hT4 plasmid DNA. After transfection, CCK-8 assay, cell cycle or protein analysis were performed at indicated time points. (A) The cell growth was monitored by CCK-8 assay for 7 days after transfection ( $\left.{ }^{*} P<0.05,{ }^{*} P<0.01\right)$. (B) The TIM-4 mRNA was detected by RT-PCR on day 7 after transfection. (C) At $48 \mathrm{~h}$ after transfection, cell cycle analysis was performed by PI staining and flow cytometry. The represented graph of each group is indicated. (D) At $48 \mathrm{~h}$ after transfection, the protein expression of PCNA, cyclin A1, B1 and D1 was analysed by western blot. These experiments were repeated at least three times.

potential role of RGD motif and integrin av $\beta 3$ signalling pathway in TIM-4-promoted lung cancer progression. First, $\alpha \mathrm{v} \beta 3$ blocking antibody was utilised to verify the possible role of $\alpha \mathrm{v} \beta 3$ in this process. As shown in Figure $4 \mathrm{~A}$, the effect of TIM-4 on the growth of A549 cells was abolished upon treatment with $\alpha \mathrm{v} \beta 3$ blocking antibody. Then, the recombinant vector pc3-hT4(m) of human TIM-4 with RGD motif mutation was constructed, and A549 cells were transfected with plasmid DNA of pc3-hT4 or pc3-hT4(m). The Co-IP results showed that integrin $\alpha \mathrm{v} \beta 3$ could interact with wild-type TIM-4 but not RGD motif-mutated TIM-4 (Figure 4B).

To elucidate whether the effects of TIM-4 on A549 cell growth, proliferation and cell cycle progression also depend on its RGD motif, A549 cells were transfected with plasmid DNA of pc3, pc3hT4 or pc3-hT4(m), and CCK-8 assay, cell cycle analysis, western blot and EdU immunofluorescence staining were performed separately at indicated time points post transfection. The results showed that the mutation of RGD motif abolished the effects of TIM-4 on growth, proliferation and cell cycle progression of A549 cells (Figures 5A-E), suggesting that TIM-4 indeed exert its function in lung cancer cells by interaction with $\alpha \mathrm{v} \beta 3$ through its RGD domain.

TIM-4 promotes lung cancer growth and proliferation in vivo. To clarify the role of TIM-4 in NSCLC in vivo, A549 cells were transplanted subcutaneously into $\mathrm{BALB} / \mathrm{c}$ nude mice.
Tumours were injected with pc3, pc3-hT4 or pc3-hT4(m) plasmids as indicated respectively. Tumour volume monitoring and weight analysis showed that the tumour growth rate of mice with pc3-hT4 intratumoural administration was significantly higher than that of the control group, whereas no significant differences were found between mice with pc3-hT4 $(\mathrm{m})$ and $\mathrm{pc} 3$ injection group (Figures $6 \mathrm{~A}-\mathrm{C}$ ). Real-time PCR (Figure 6D) and IHC staining (Figures 7A and $\mathrm{B}$ ) results showed higher level expression of TIM-4 in tumours from mice with pc3-hT4 and pc3-hT4(m) administration than the control group, indicating successful overexpression of TIM-4 in tumours from these two groups. Ki-67 is a well-known marker for cell proliferation (Tabata et al, 2014). In this study, higher expression of Ki-67 was observed in tumours from mice with pc3hT4 administration, but not in pc3-hT4(m) plasmid DNA administration group (Figures $7 \mathrm{C}$ and $\mathrm{D}$ ). These results further proved that TIM-4 promoted lung cancer growth and proliferation by its RGD motif.

\section{DISCUSSION}

In this study, we describe for the first time an important role of TIM-4 in NSCLC. The augmented expression of TIM-4 in NSCLC cancer tissues is identified. Importantly, higher expression 

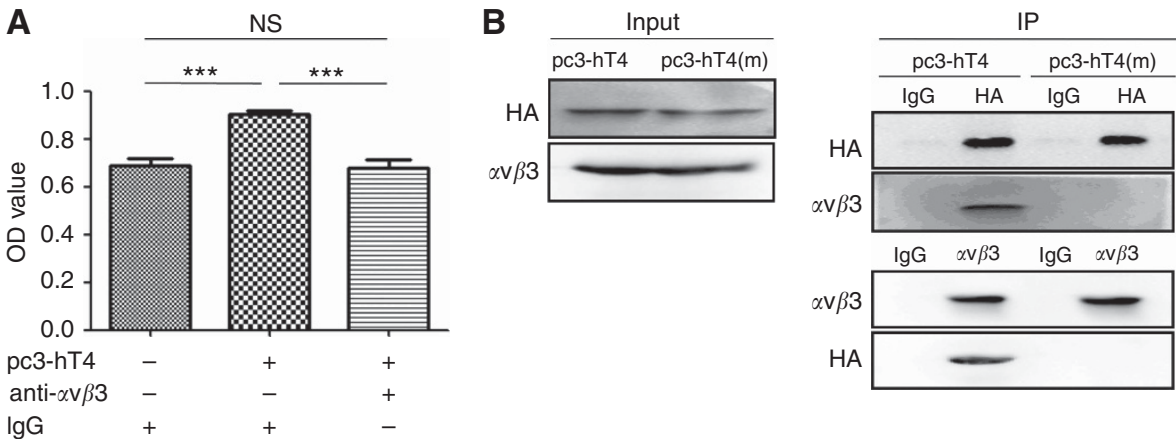

Figure 4. T-cell immunoglobulin domain and mucin domain 4 (TIM-4) interacts with integrin $\alpha \mathrm{v} \beta 3$ through its RGD motif. The A549 cells were seeded in 96- or 6-well plates. After grown to 80-90\% confluence, the cells were transfected with pc3, pc3-hT4 or pc3-hT4(m) plasmid DNA separately. After transfection, $\alpha v \beta 3$ blocking or Co-IP was performed at indicated time points. (A) At $6 \mathrm{~h}$ after transfection, $25 \mu \mathrm{g} \mathrm{ml}^{-1}$ of anti- $\alpha \mathrm{v} \beta 3$ or mouse IgG was added into cells and incubated for 4 days. The cell growth was monitored by CCK-8 assay $\left({ }^{\star \star \star} \mathrm{P}<0.001\right)$. (B) At $48 \mathrm{~h}$ after transfection with pc3-hT4 or pc3-hT4 $(\mathrm{m})$ plasmid DNA, Co-IP assay was performed. These experiments were repeated at least three times.

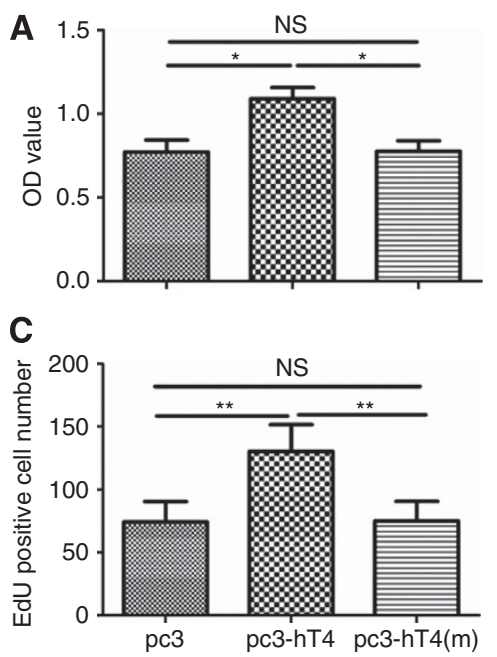

D

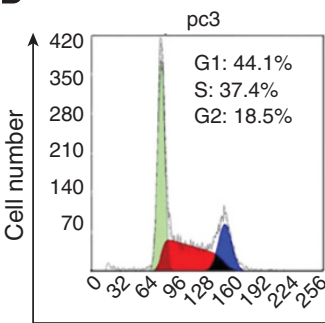

B
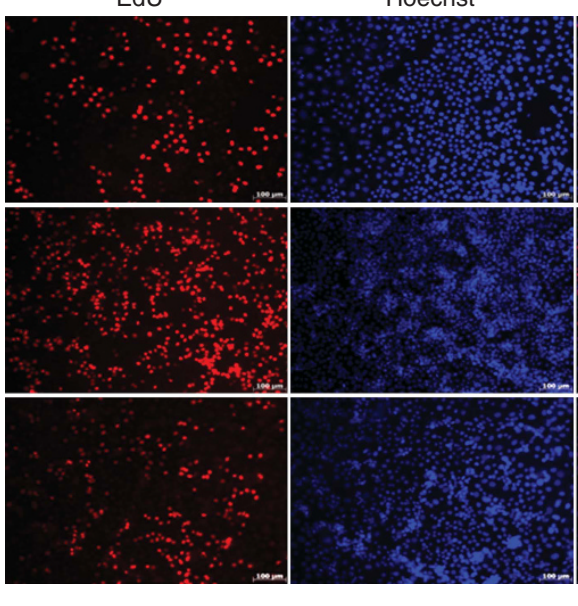

E

Hoechst
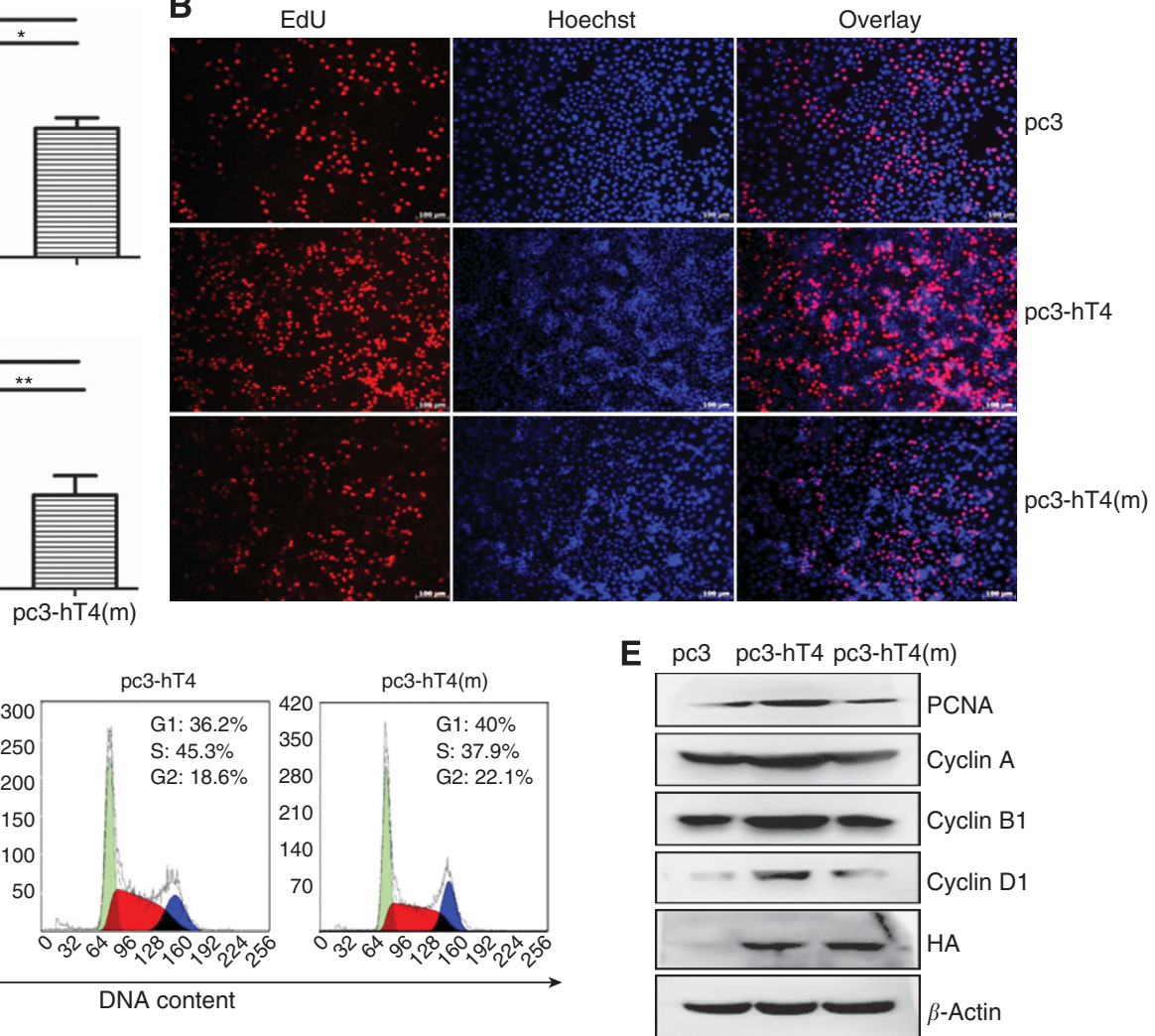

DNA content

Figure 5. T-cell immunoglobulin domain and mucin domain 4 (TIM-4) promotes lung cancer cell growth and proliferation by its RGD motif. The A549 cells were seeded in 96- or 6-well plates. After grown to 80-90\% confluence, the cells were transfected with pc3, pc3-hT4 or pc3-hT4(m) plasmid DNA separately. After transfection, CCK-8 assay, EdU staining, cell cycle or protein analysis was performed at indicated time points. (A) At 5 days after transfection, the cell growth was monitored by CCK-8 assay ( $\left.{ }^{\star} P<0.05\right)$. (B and C) At $94 \mathrm{~h}$ after transfection, cell proliferation was assayed by EdU staining and fluorescence microscope. Scale bars, $100 \mu \mathrm{m}\left({ }^{\star *} \mathrm{P}<0.01\right.$ ). (D) At $48 \mathrm{~h}$ after transfection, the cell cycle was analysed by $\mathrm{PI}$ staining and flow cytometry. The represented graph of each group is indicated. (E) At $48 \mathrm{~h}$ after transfection, the protein expression of PCNA, cyclin A1, B1 and D1 was analysed by western blot.

of TIM-4 in lung cancer tissues indicates poor prognosis of patients. Stimulation of LPS and cytokine increases TIM-4 expression in A549 cells. We further show that overexpression of TIM-4 in A549 cells promotes lung cancer cell growth and proliferation both in vitro and in vivo. Our study strongly suggests that TIM-4 is essential for lung cancer progression. The TIM-4 interacts with integrin $\alpha \mathrm{v} \beta 3$ through its RGD motif in IgV domain. Furthermore, we provide evidence that TIM- 4 promotes lung cancer growth and proliferation depending on RGD domain, suggesting that TIM-4 might be a potential diagnostic marker and therapeutic target of lung carcinoma.

The TIM-4, also named TIMD4, is a cell-surface glycoprotein belonging to TIM family. As a co-stimulator and natural ligand of TIM-1, TIM-4 is involved in specific immune responses required for T-cell activation (Rodriguez-Manzanet et al, 2008; Mizui et al, 2008). As a receptor for phosphatidylserine, TIM-4 controls 

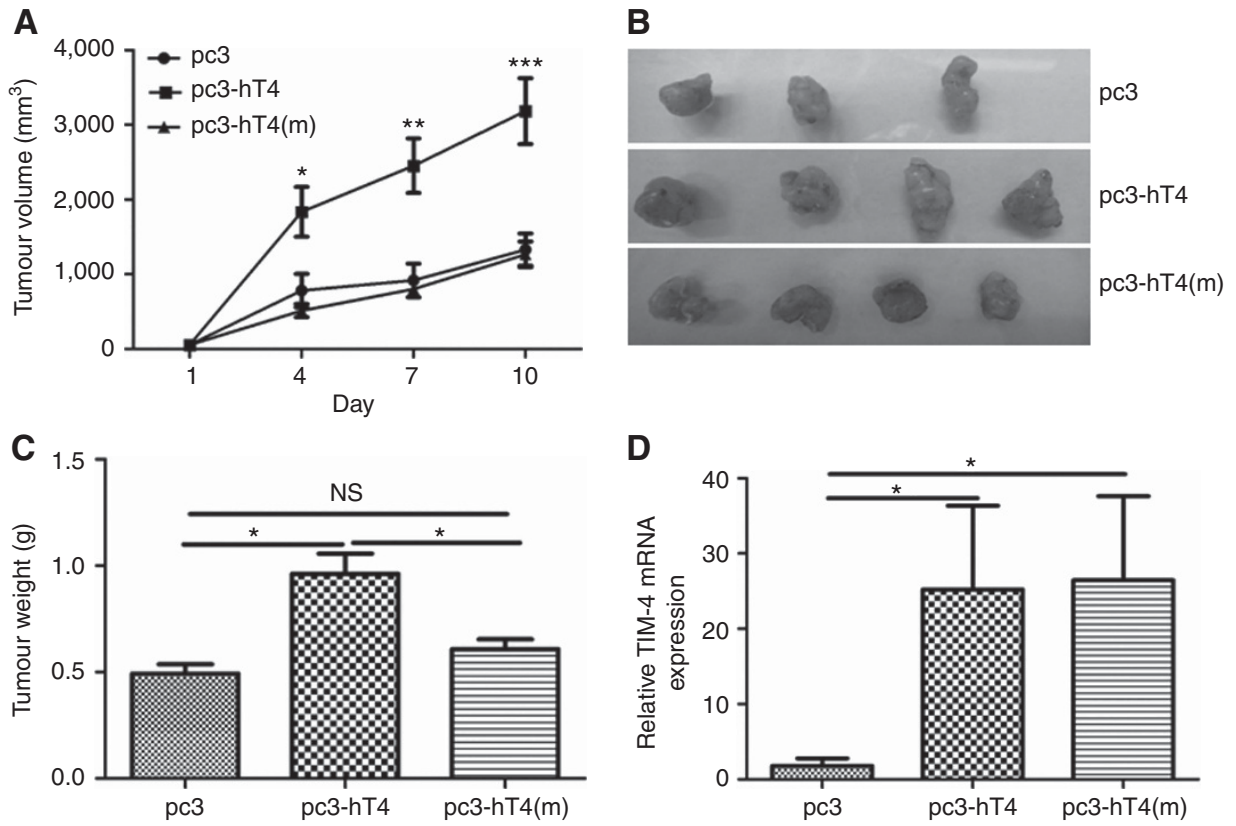

Figure 6. T-cell immunoglobulin domain and mucin domain 4 (TIM-4) promotes lung cancer growth in vivo. The $5 \times 10^{7}$ A549 cells in $100 \mu$ I PBS were subcutaneously injected into the left axillae of the nude mice. After reaching a diameter of $0.5 \mathrm{~cm}$, the tumours were injected with plasmid DNA (20 $\mu \mathrm{g}$ per $100 \mu \mathrm{l}$ in PBS) of pc3, pc3-hT4 or pc3-hT4(m) every fourth day for a total of 4 injections. (A) The tumour size was monitored every other day. Tumour volume was calculated using the following formula: volume $=$ width $^{2} \times$ length $\times 1 / 2$ and growth curve was drawn subsequently $\left({ }^{\star} P<0.05,{ }^{*} P<0.01,{ }^{* \star *} P<0.001\right)$. (B and $\left.\mathbf{C}\right)$ After 14 days, the mice were killed and the tumours were isolated and weighed. Tumour images were observed $\left({ }^{\star} P<0.05\right)$. (D) The TIM-4 mRNA expression in xenograft tumour tissues was examined by real-time PCR ( $\left.P<0.05\right)$. Animal experiments were repeated twice and at least three mice were included in each group.
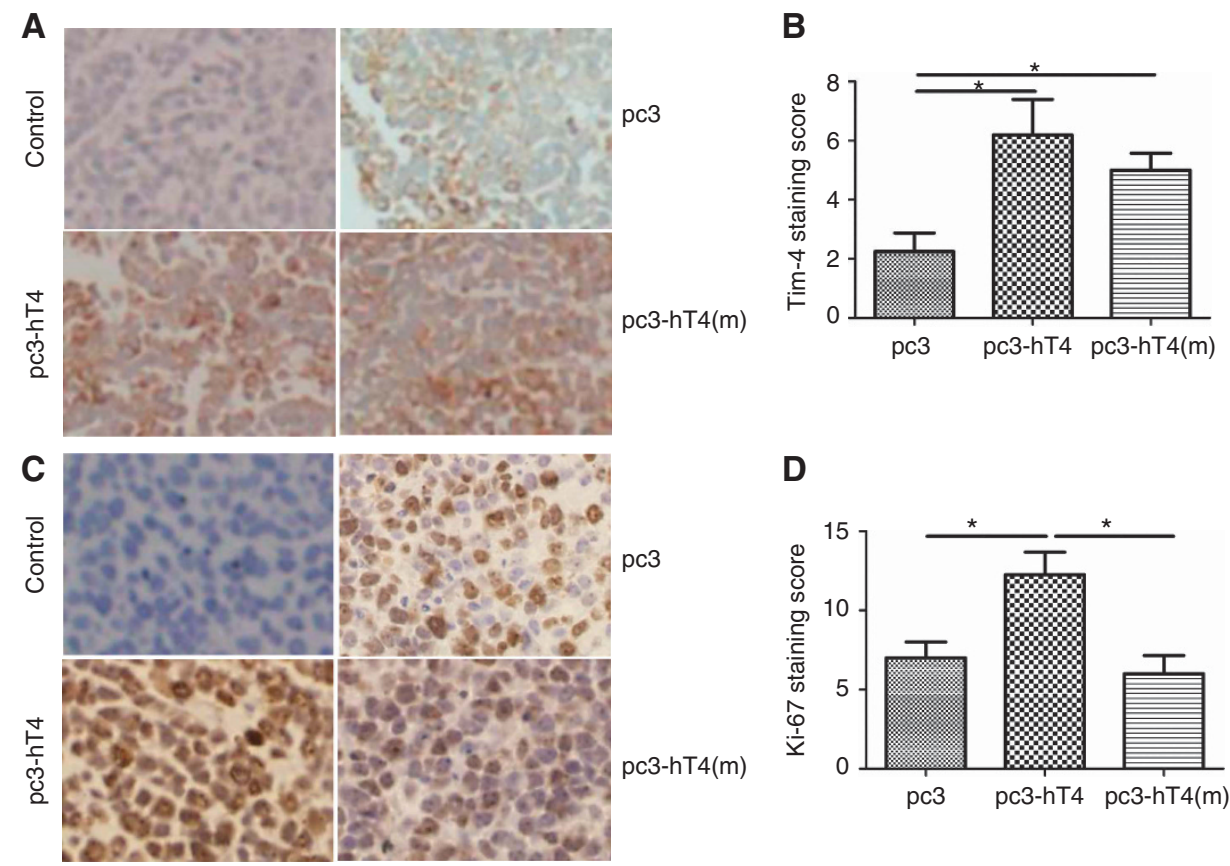

Figure 7. T-cell immunoglobulin domain and mucin domain 4 (TIM-4) and Ki-67 expression in xenograft tumour tissues. The A549 cells were subcutaneously injected into the left axillae of the nude mice, and the tumours were injected with plasmid DNA of pc3, pc3-hT4 or pc3-hT4(m) every fourth day for a total of 4 injections. After 14 days, the mice were killed and the tumours tissues fixed in 10\% buffered formalin were embedded in paraffin for IHC staining. The control represented the isotype control lgG instead of TIM-4 antibody or Ki-67 antibody in the process of IHC staining. (A) The TIM-4 and (C) Ki-67 protein expression in xenograft tumour tissues was examined by IHC staining. Photos of IHC staining are representative of at least 10 similar observations $(\times 200)$. The expression rate and staining intensity of (B) TIM-4 and (D) Ki-67 were reported separately according to the German semiquantitative scoring system $\left({ }^{\star} P<0.05\right)$.

adaptive immunity by mediating the removal of antigen-specific $\mathrm{T}$ cells (Albacker et al, 2010, 2013). Recently, studies have indicated that TIM-4 is also closely associated with tumour tolerance events.
It is reported that macrophage-derived TIM-4 may play an important role in tumour tolerance by inducing Tregs in gliomas (Xu et al, 2011). In addition, TIM-4 activates autophagy-mediated 
degradation of ingested tumours by directly interacting with AMPK $\alpha 1$ that leads to impaired CTL responses (Baghdadi et al, 2013). Intriguingly, TIM-4 is recently detected in several kinds of tumour tissues including parapharyngeal liposarcoma. Consistently, we find high expression of TIM-4 in multiple organ tumour tissue microarray and lung cancer tissues in this study. To our surprise, the intensity of TIM-4 transcripts in most of the lung cancer cell lines is low. It is well known that TIM-4 expression is highly restricted to professional APCs, specifically in macrophages and dendritic cells, but not in T cells (Meyers et al, 2005; Wong et al, 2010). Our previous studies also show high expression of TIM-4 in macrophages (Xu et al, 2010, 2015). We speculate that some factors inhibiting TIM-4 transcripts may exist in the lung cancer cell lines. For example, ZHX2 suppresses TIM-4 transcripts in mouse liver tissues (Gargalovic et al, 2010). In addition, we found that TIM-4 transcript was inducible in macrophages (Xu et al, 2010, 2015) as well as lung cancer cells (Supplementary Figures $2 \mathrm{~B}$ and $\mathrm{C}$ ). Normally, lung cancer cells produce low levels of these cytokines that might contribute to low TIM- 4 transcripts in lung cancer cell lines. Interestingly, we demonstrate TIM-4 expression in A549 and NCI-H1975 cells is increased by stimulations with LPS, IL- 6, TNF- $\alpha$ or TGF- $\beta$. High levels of LPS or these cytokines exist in local lung cancer tissues (Nathan and Ding, 2010; Melkamu et al, 2013), indicating that the tumour microenvironments might induce TIM-4 expression, consistent with high expression of TIM-4 in human NSCLC tissues in our study and enhanced expression of TIM- 4 on tumour-associated myeloid cells induced by damage-associated molecular patterns from tumour cells (Baghdadi et al, 2013). However, whether TIM-4 expressed in tumour cells is involved in inflammation-mediated tumourigenesis requires to be further investigated.

The major finding of our investigation is the essential role of TIM-4 in the maintenance of lung cancer growth. Strikingly, the use of FG-CC' siRNA blocking interaction of TIM-1 and TIM-4 is capable of enhancing DC vaccine against gastric cancer (Sun et al, 2012). Combined blockade of TIM-3 and TIM-4 augments cancer vaccine efficacy against established melanomas (Baghdadi et al, 2013). Our group and other researchers have found that the ectopic expression of TIM-3 in tumour cells may be a potentially prognostic marker for patients with NSCLC, prostate cancer, clear cell renal carcinoma or cervical cancer (Zhuang et al, 2012; Cao et al, 2013; Piao et al, 2013; Yuan et al, 2014). However, the role of TIM-4 in tumour cells is undefined. In this study, we confirm that ectopic expression of TIM-4 in NSCLC is a potential marker of poor differentiation and short lifespan of patients. More importantly, overexpression of TIM-4 promotes A549 cell growth and proliferation both in vitro and in vivo. These data further elucidate the critical role of TIM-4 in NSCLC progression. It has been found that targeting of the TIM-4-AMPK $\alpha 1$ interaction constitutes a unique strategy for augmenting antitumour immunity and improving cancer chemotherapy (Baghdadi et al, 2013). Moreover, TIM-4 knockdown in A549 cells inhibits the growth of cells (Supplementary Figure 3). Therefore, targeting TIM-4 might be a hopeful way to eradicate tumour by inhibiting tumour growth and improving immune tolerance cooperatively.

The molecular mechanisms underlying TIM-4-mediated cancer progression remain completely unknown. However, TIM-4 does not mediate direct signalling because of lack of tyrosine phosphorylation site in cytoplasmic tail (Park et al, 2009). Different from other TIM proteins, TIM- 4 contains an RGD motif in its IgV domain that is a hallmark for adhesive proteins (RodriguezManzanet et al, 2009). The RGD sequence is recognised by the integrin family of cell-surface receptors, and RGD peptides preferentially bind to the $\alpha \mathrm{v} \beta 3$ integrin (Ruoslahti, 1996; Danhier et al, 2012). In this study, the interaction of TIM- 4 with $\alpha \mathrm{v} \beta 3$ is verified by Co-IP assay. Moreover, RGD motif is responsible for the tumourigenic potential of TIM-4 in lung cancer. This finding is consistent with the role of molecules containing RGD motif, such as OPN, Del-1 and MFG-E8, in tumour growth (Aoka et al, 2002; Cui et al, 2007; Carrascosa et al, 2012). We have proved that TIM-4 interacts with integrin $\alpha \mathrm{v} \beta 3$ through its RGD motif in IgV domain. The RGD motif and integrin av $\beta 3$ signalling pathway are responsible for TIM-4-promoted lung cancer progression. However, the interaction of TIM- 4 with $\alpha 5 \beta 1$ or other integrins could not be excluded. Very recently, it is reported that TIM-4 utilises integrins as co-receptors to effect phagocytosis of apoptotic cells with the participation of Src-family kinases, FAK, phosphatidylinositol 3,4,5-trisphosphate, nucleotide-exchange factor Vav3 as well as Rho-family GTPases (Flannagan et al, 2014). Furthermore, RGD-peptide lunasin inhibits Akt-mediated NF- $\kappa$ B pathway by interaction with the $\alpha \mathrm{v} \beta 3$ integrin (Cam and de Mejia, 2012). In addition, depletion of endothelial $\beta 3$-integrin transiently inhibits tumour growth and angiogenesis in mice (Steri et al, 2014). However, whether Src, Akt signalling pathway or vessel formation are involved in TIM-4-mediated lung cancer progression requires to be explored more in depth in the future. Apoptosis signal pathway might also be involved in the process of TIM-4-promoted lung cancer progression as overexpression of TIM-4 inhibits apoptosis of lung cancer cells (Supplementary Figure 4).

In summary, our results suggest that TIM- 4 might be a potential biomarker for NSCLC. We initially demonstrate the underlying molecular mechanisms that TIM-4 promotes growth and proliferation of lung cancer cells by its RGD motif. Our findings provide new insight into the lung cancer pathogenesis mediated by TIM- 4 and a novel candidate target for the effective therapy of lung cancer.

\section{ACKNOWLEDGEMENTS}

We gratefully acknowledge the helpful suggestions of Lei Lei from the NIH. This work was supported in part by grants from the National Nature Science Foundation of China (nos. 30872298, 81172862 and 81100203), Research Fund for the Doctoral Program of Higher Education of China (RFDP) (20110131110034) and the Shandong Provincial Natural Science Foundation (no. ZR2010HM025).

\section{CONFLICT OF INTEREST}

The authors declare no conflict of interest.

\section{REFERENCES}

Abe Y, Kamachi F, Kawamoto T, Makino F, Ito J, Kojima Y, Moustapha Ael D, Usui Y, Yagita H, Takasaki Y, Okumura K, Akiba H (2013) TIM-4 has dual function in the induction and effector phases of murine arthritis. J Immunol 191(9): 4562-4572.

Albacker LA, Karisola P, Chang YJ, Umetsu SE, Zhou M, Akbari O, Kobayashi N, Baumgarth N, Freeman GJ, Umetsu DT, DeKruyff RH (2010) TIM-4, a receptor for phosphatidylserine, controls adaptive immunity by regulating the removal of antigen-specific T cells. J Immunol 185(11): 6839-6849.

Albacker LA, Yu S, Bedoret D, Lee WL, Umetsu SE, Monahan S, Freeman GJ, Umetsu DT, DeKruyff RH (2013) TIM-4, expressed by medullary macrophages, regulates respiratory tolerance by mediating phagocytosis of antigen-specific T cells. Mucosal Immunol 6(3): 580-590.

Aoka Y, Johnson FL, Penta K, Hirata KiK, Hidai C, Schatzman R, Varner JA, Quertermous T (2002) The embryonic angiogenic factor Dell accelerates tumor growth by enhancing vascular formation. Microvasc Res 64(1): 148-161. Baghdadi M, Nagao H, Yoshiyama H, Akiba H, Yagita H, Dosaka-Akita H, Jinushi M (2013) Combined blockade of TIM-3 and TIM-4 augments cancer vaccine efficacy against established melanomas. Cancer Immunol Immunother 62(4): 629-637.

Baghdadi M, Yoneda A, Yamashina T, Nagao H, Komohara Y, Nagai S, Akiba H, Foretz M, Yoshiyama H, Kinoshita I, Dosaka-Akita H, Takeya M, Viollet B, 
Yagita H, Jinushi M (2013) TIM-4 glycoprotein-mediated degradation of dying tumor cells by autophagy leads to reduced antigen presentation and increased immune tolerance. Immunity 39(6): 1070-1081.

Boland JM, Kwon ED, Harrington SM, Wampfler JA, Tang H, Yang P, Aubry MC (2013) Tumor B7-H1 and B7-H3 expression in squamous cell carcinoma of the lung. Clin Lung Cancer 14(2): 157-163.

Cam A, de Mejia EG (2012) RGD-peptide lunasin inhibits Akt-mediated NF$\kappa \mathrm{B}$ activation in human macrophages through interaction with the $\alpha \mathrm{V} \beta 3$ integrin. Mol Nutr Food Res 56(10): 1569-1581.

Cao Y, Zhou X, Huang X, Li Q, Gao L, Jiang L, Huang M, Zhou J (2013) Tim3 expression in cervical cancer promotes tumor metastasis. PLoS One 8(1): e53834.

Carrascosa C, Obula RG, Missiaglia E, Lehr HA, Delorenzi M, Frattini M, Rüegg C, Mariotti A (2012) MFG-E8/lactadherin regulates cyclins D1/D3 expression and enhances the tumorigenic potential of mammary epithelial cells. Oncogene 31(12): 1521-1532.

Chen C, Qu QX, Shen Y, Mu CY, Zhu YB, Zhang XG, Huang JA (2012) Induced expression of $\mathrm{B} 7-\mathrm{H} 4$ on the surface of lung cancer cell by the tumor-associated macrophages: a potential mechanism of immune escape. Cancer Lett 317(1): 99-105.

Cui R, Takahashi F, Ohashi R, Gu T, Yoshioka M, Nishio K, Ohe Y, Tominaga S, Takagi Y, Sasaki S, Fukuchi Y, Takahashi K (2007) Abrogation of the interaction between osteopontin and alphavbeta3 integrin reduces tumor growth of human lung cancer cells in mice. Lung Cancer 57(3): 302-310.

Danhier F, Le Breton A, Préat V (2012) RGD-based strategies to target alpha(v) beta(3) integrin in cancer therapy and diagnosis. Mol Pharm 9(11): 2961-2973.

Dorfman DM, Hornick JL, Shahsafaei A, Freeman GJ (2010) The phosphatidylserine receptors, $\mathrm{T}$ cell immunoglobulin mucin proteins 3 and 4, are markers of histiocytic sarcoma and other histiocytic and dendritic cell neoplasms. Hum Pathol 41(10): 1486-1494.

Flannagan RS, Canton J, Furuya W, Glogauer M, Grinstein S (2014) The phosphatidylserine receptor TIM4 utilizes integrins as coreceptors to effect phagocytosis. Mol Biol Cell 25(9): 1511-1522.

Gargalovic PS, Erbilgin A, Kohannim O, Pagnon J, Wang X, Castellani L, LeBoeuf R, Peterson ML, Spear BT, Lusis AJ (2010) Quantitative trait locus mapping and identification of ZHX2 as a novel regulator of plasma lipid metabolism. Circ Cardiovasc Genet 3(1): 60-67.

Goc A, Liu J, Byzova TV, Somanath PR (2012) Akt1 mediates prostate cancer cell microinvasion and chemotaxis to metastatic stimuli via integrin $\beta_{3}$ affinity modulation. Br J Cancer 107(4): 713-723.

Han CP, Kok LF, Wang PH, Wu TS, Tyan YS, Cheng YW, Lee MY, Yang SF (2009) Scoring of p16(INK4a) immunohistochemistry based on independent nuclear staining alone can sufficiently distinguish between endocervical and endometrial adenocarcinomas in a tissue microarray study. Mod Pathol 22(6): 797-806.

Kim HS, Kim HS, Lee CW, Chung DH (2010) T cell Ig domain and mucin domain 1 engagement on invariant NKT cells in the presence of TCR stimulation enhances IL-4 production but inhibits IFN-gamma production. J Immunol 184(8): 4095-4106.

Li H, Zhou X, Ran Q, Wang L (2013a) Parapharyngeal liposarcoma: a case report. Diagn Pathol 8: 42.

Li J, Cao D, Guo G, Wu Y, Chen Y (2013b) Expression and anatomical distribution of TIM-containing molecules in Langerhans cell sarcoma. $J$ Mol Histol 44(2): 213-220.

Li Z, Ju Z, Frieri M (2013c) The T-cell immunoglobulin and mucin domain (Tim) gene family in asthma, allergy, and autoimmunity. Allergy Asthma Proc 34(1): e21-e26.

Melkamu T, Qian X, Upadhyaya P, O'Sullivan MG, Kassie F (2013) Lipopolysaccharide enhances mouse lung tumorigenesis: a model for inflammation-driven lung cancer. Vet Pathol 50(5): 895-902.

Meyers JH, Chakravarti S, Schlesinger D, Illes Z, Waldner H, Umetsu SE, Kenny J, Zheng XX, Umetsu DT, DeKruyff RH, Strom TB, Kuchroo VK (2005) TIM-4 is the ligand for TIM-1, and the TIM-1-TIM-4 interaction regulates T cell proliferation. Nat Immunol 6(5): 455-464.

Mizui M, Shikina T, Arase H, Suzuki K, Yasui T, Rennert PD, Kumanogoh A, Kikutani H (2008) Bimodal regulation of T cell-mediated immune responses by TIM-4. Int Immunol 20(5): 695-708.

Nathan C, Ding A (2010) Nonresolving inflammation. Cell 140(6): 871-882.
Park D, Hochreiter-Hufford A, Ravichandran KS (2009) The phosphatidylserine receptor TIM-4 does not mediate direct signaling. Curr Biol 19(4): 346-351.

Piao YR, Piao LZ, Zhu LH, Jin ZH, Dong XZ (2013) Prognostic value of T cell immunoglobulin mucin-3 in prostate cancer. Asian Pac J Cancer Prev 14(6): 3897-3901.

Ramaekers F, Broers J, Rot MK, Oostendorp T, Wagenaar S, Vooijs P (1987) Detection of epithelial- and neural type of intermediate filament proteins in human lung tumors. Acta Histochem Suppl 34: 45-56.

Rodriguez-Manzanet R, DeKruyff R, Kuchroo VK, Umetsu DT (2009) The costimulatory role of TIM molecules. Immunol Rev 229(1): 259-270.

Rodriguez-Manzanet R, Meyers JH, Balasubramanian S, Slavik J, Kassam N, Dardalhon V, Greenfield EA, Anderson AC, Sobel RA, Hafler DA, Strom TB, Kuchroo VK (2008) TIM-4 expressed on APCs induces T cell expansion and survival. J Immunol 180(7): 4706-4713.

Ruoslahti E (1996) RGD and other recognition sequences for integrins. Annu Rev Cell Dev Biol 12: 697-715.

Siegel R, Ma J, Zou Z, Jemal A (2014) Cancer statistics 2014. CA Cancer J Clin 64(1): 9-29.

Steri V, Ellison TS, Gontarczyk AM, Weilbaecher K, Schneider JG, Edwards D, Fruttiger M, Hodivala-Dilke KM, Robinson SD (2014) Acute depletion of endothelial $\beta 3$-integrin transiently inhibits tumor growth and angiogenesis in mice. Circ Res 114(1): 79-91.

Sun HW, Wu C, Tan HY, Wang QS (2012) A new development of FG-CC' siRNA blocking interaction of Tim-1 and Tim- 4 can enhance DC vaccine against gastric cancer. Hepatogastroenterology 59(120): 2677-2682.

Tabata K, Tanaka T, Hayashi T, Hori T, Nunomura S, Yonezawa S, Fukuoka J (2014) Ki-67 is a strong prognostic marker of non-small cell lung cancer when tissue heterogeneity is considered. BMC Clin Pathol 14: 23.

Tian M, Neil JR, Schiemann WP (2011) Transforming growth factor- $\beta$ and the hallmarks of cancer. Cell Signal 23(6): 951-962.

Toda S, Hanayama R, Nagata S (2012) Two-step engulfment of apoptotic cells. Mol Cell Biol 32(1): 118-125.

Velcheti V, Schalper KA, Carvajal DE, Anagnostou VK, Syrigos KN, Sznol M, Herbst RS, Gettinger SN, Chen L, Rimm DL (2014) Programmed death ligand-1 expression in non-small cell lung cancer. Lab Invest 94(1): $107-116$.

Wong K, Valdez PA, Tan C, Yeh S, Hongo JA, Ouyang W (2010) Phosphatidylserine receptor Tim- 4 is essential for the maintenance of the homeostatic state of resident peritoneal macrophages. Proc Natl Acad Sci USA 107(19): 8712-8717.

Xu LY, Qi JN, Liu X, Ma HX, Yuan W, Zhao PQ, Liang XH, Xu Y, Wang HX, Xu XY, Wang W, Ma CH, Gao LF (2015) Tim-4 inhibits NO generation by murine macrophages. PLoS One 10(4): e0124771.

Xu L, Qi J, Zhao P, Liang X, Ju Y, Liu P, Liu B, Guo C, Zhang L, Ma C, Gao L (2010) T cell immunoglobulin- and mucin-domain-containing molecule-4 attenuates concanavalin A-induced hepatitis by regulating macrophage. J Leukoc Biol 88(2): 329-336.

Xu L, Xiao H, Xu M, Zhou C, Yi L, Liang H (2011) Glioma-derived T cell immunoglobulin- and mucin domain-containing molecule-4 (TIM4) contributes to tumor tolerance. J Biol Chem 286(42): 36694-36699.

Yuan J, Jiang B, Zhao H, Huang Q (2014) Prognostic implication of TIM-3 in clear cell renal cell carcinoma. Neoplasma 61(1): 35-40.

Zhao P, Xu L, Wang P, Liang X, Qi J, Liu P, Guo C, Zhang L, Ma C, Gao L (2010) Increased expression of human T-cell immunoglobulin- and mucin-domain-containing molecule-4 in peripheral blood mononuclear cells from patients with system lupus erythematosus. Cell Mol Immunol 7(2): 152-156

Zhuang X, Zhang X, Xia X, Zhang C, Liang X, Gao L, Zhang X, Ma C (2012) Ectopic expression of TIM-3 in lung cancers: a potential independent prognostic factor for patients with NSCLC. Am J Clin Pathol 137(6): 978-985.

This work is published under the standard license to publish agreement. After 12 months the work will become freely available and the license terms will switch to a Creative Commons AttributionNonCommercial-Share Alike 4.0 Unported License.

Supplementary Information accompanies this paper on British Journal of Cancer website (http://www.nature.com/bjc) 